\title{
Sophisticated test facility to detect land mines
}

\author{
Wim de Jong, Henk A. Lensen, Yvonne H.L. Janssen
}

TNO Physics and Electronics Laboratory, PO Box 96864, 2509 JG The Hague, The Netherlands

\begin{abstract}
In the framework of the Dutch government humanitarian demining project 'HOM-2000', an outdoor test facility has been realized to test, improve and develop detection equipment for land mines. This sophisticated facility, allows us to access and compare the performance of the individual and of a combination of different sensors (sensor fusion) against a variety of threats. The test facility entails six test lanes of 30 square meters each, filled with different types of soil. The groundwater level of the lanes can be regulated separately and the temperature of the soil and of the test mines is monitored.

A moveable measurement platform has been realized which is completely free of electrically conducting materials. With this platform the individual or fused detection systems can be moved automatically over the whole test site with an accuracy of one centimeter in every direction.

Test mines and minelike objects have been placed in the lanes. The set of test mines contains nine different types of both anti personnel and anti tank mines, low metal content and non-metal mines. To simulate the high explosives, the test mines have been filled with a silicone rubber.
\end{abstract}

Keywords: complete test facility, different soils, test mines, measurement platform, controlled conditions

\section{INTRODUCTION}

The use of multi-sensor technology has become part of a growing number of research programs and projects that seek to develop more efficient and reliable mine detection techniques. The 'Humanitarian Demining 2000' (HOM-2000) project is a Dutch government initiative focusing on the development of both a hand carried and a vehicle mounted mine detection systems that achieve improved performance through simultaneous use of metal detection (MD), ground penetrating radar (GPR) and thermal infrared imaging (TIR). Various sensor combinations and sensor fusion options have been examined in order to access the feasibility of the HOM-2000 objectives. An outdoor test facility is situated on the proving ground "Waalsdorp" near TNO Physics and Electronics Laboratory (TNO-FEL) in the Netherlands, (4.33" eastern longitude, $52.11^{\circ}$ northern latitude).

This paper describes the test facility, which is shown in Figure 1, and is organized as follows. Section 2 describes the construction of the test lanes and the characteristics of the soils in these lanes. Section 3 gives a description of the measurement platform. The test mines and their properties are described in section 4 . Section 5 deals with additional equipment which is realized and gives results obtained with this equipment. In the finishing section 6 some conclusions are drawn. Due to security reasons, some details have been omitted in this paper. 


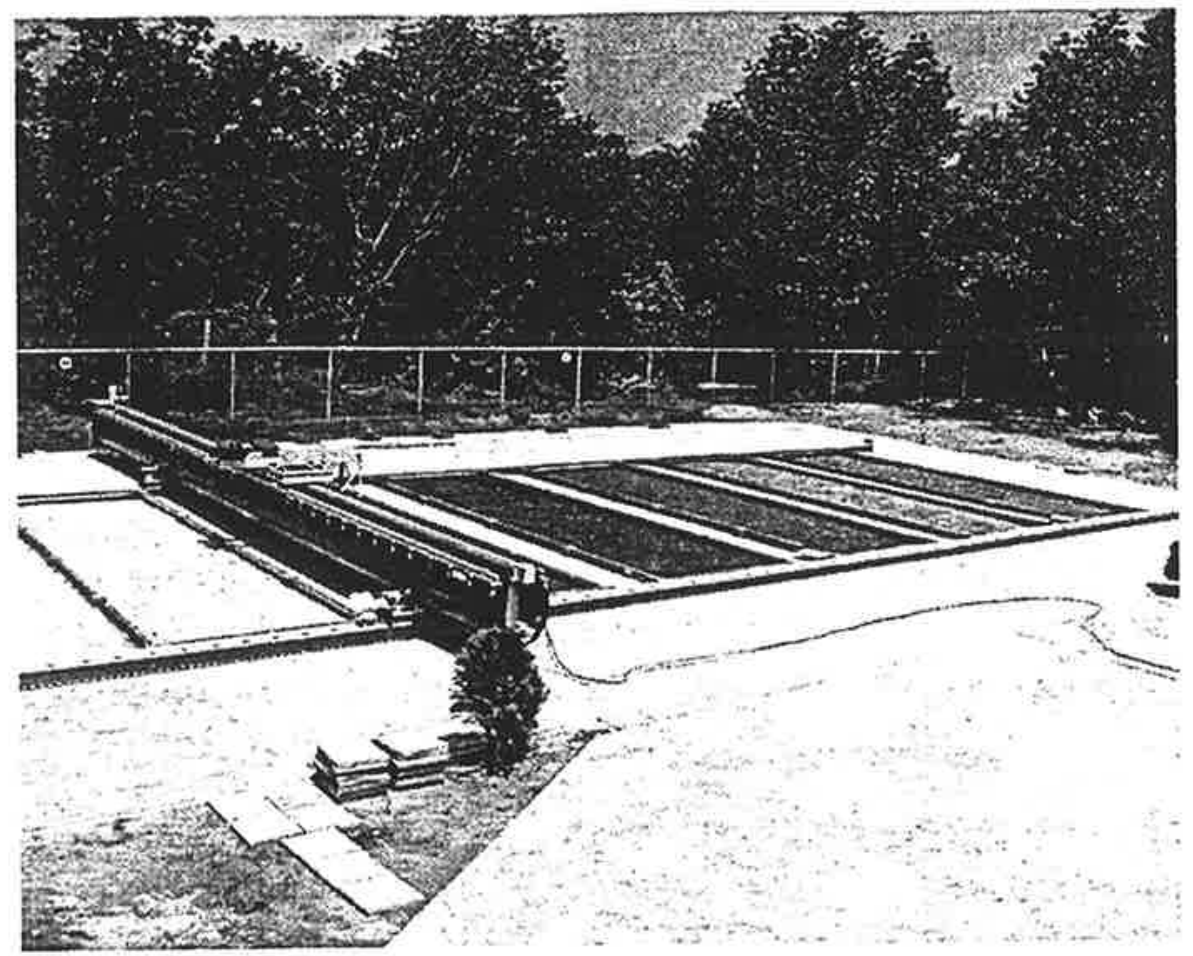

Figure 1. Overview of the six test lanes (each 10 meters long and 3 meters wide) and the measurement platform. Figure 2 and Figure 3 show details af the lanes and the measurement platform.

\section{TESTLANES}

In total six lanes have been constructed with a different soil type in each lane. Figure 2 gives a schematic drawing of the layout. The dimensions of the test lanes are $10 \mathrm{~m} \mathrm{x} 3 \mathrm{~m} \mathrm{x} 1.5 \mathrm{~m}$ for length, width and depth, with a distance of $1 \mathrm{~m}$ between them. In order to determine the dimensions, the following considerations have been taken into account. The minimal width of the lanes is based on a vehicle mounted detection system with a maximal width of $1 \mathrm{~m}$. Based on this width and to avoid edge effects a width of $3 \mathrm{~m}$ was chosen. The depth of $1.5 \mathrm{~m}$ was chosen to keep the soil profile as natural as possible. Disturbing radar reflections from the bottom of the lanes are expected to be minimal at this depth. To put as much different mines in the lanes as possible, and to be able to measure with a forward looking IR system, a length of $10 \mathrm{~m}$ was chosen. The distance of $1 \mathrm{~m}$ between the lanes is sufficient to prevent the disturbance of objects situated in the adjacent lanes. To avoid distortion of the MD measurements the lanes have been constructed of wood without the use of electrically conducting engineering materials. Around the 6 lanes a zone of $5 \mathrm{~m}$ wide and $1.5 \mathrm{~m}$ deep is made free of metals. 


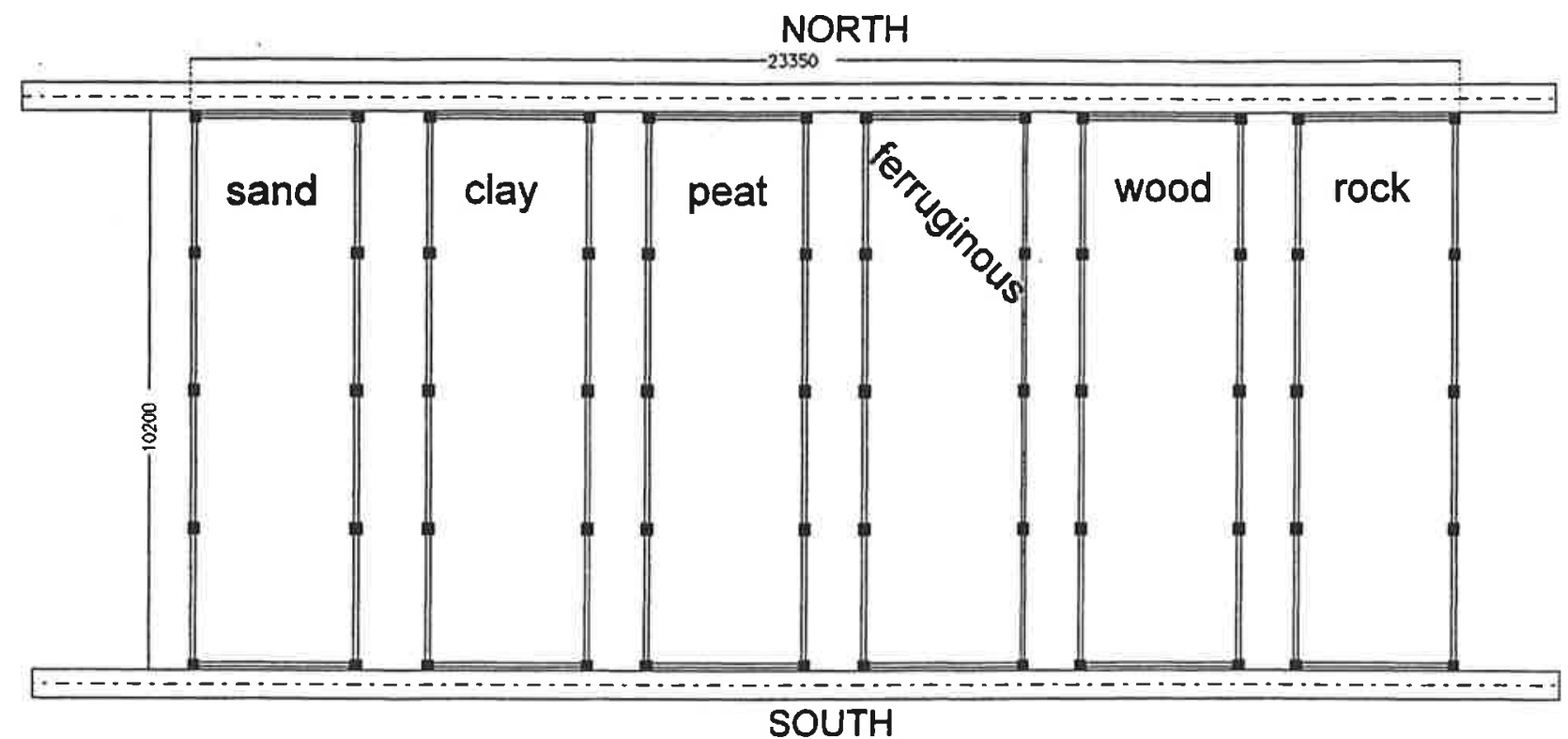

Figure 2. Layout of the test facility. The measures in this drawing are in millimeters. The horizontal bars above and below the 6 lanes give the position for the rails of the measurement platform (section 3). The properties of the soils are given in Table 1.

\subsection{SOIL TYPES}

Land mines can be found in different soil types like clay, sand, stones, rock etc. Preferably native soils from countries with a large mine threat should be taken. However due to environmental regulations in the Netherlands, only native soils from the Netherlands were allowed to fill the lanes with. A limited number of soils has been chosen that covers a wide range of different soils. The test lanes were filled with the following soil types: sandy soil, clay, peat, ferruginous soil, a sandy woodland soil (partly with roots and partly without roots) and a sandy soil with a variety of small and large rocks. For the first five types of soil a chemical and physical description is available which gives insight of the texture and the structure of the different types of soil. While filling the lanes, the natural layer structure of the soil was preserved.

Table 1. Typical soil characteristics of the first $50 \mathrm{~cm}$. Iron, organic matter and lutum content are given relative to the dry matter content. The last column gives the typical groundwater level.

\begin{tabular}{|l|c|c|c|c|c|}
\hline Soil & $\begin{array}{c}\text { Dry matter } \\
\text { density } \\
\left(\mathrm{kg} / \mathrm{m}^{3}\right)\end{array}$ & $\begin{array}{c}\text { Iron content } \\
(\mathrm{g} / \mathrm{kg})\end{array}$ & $\begin{array}{c}\text { Organic matter } \\
\text { content }\end{array}$ & $\begin{array}{c}\text { Lutum } \\
\text { content }\end{array}$ & $\begin{array}{c}\text { Groundwater } \\
\text { level }\end{array}$ \\
\hline Sand & 1525 & 1.9 & $<1 \%$ & $<2 \%$ & $120 \mathrm{~cm}$ \\
\hline Clay & 1422 & 22 & $3 \%$ & $27 \%$ & $120 \mathrm{~cm}$ \\
\hline Peat & 943 & 39 & $35 \%$ & $15 \%$ & $40 \mathrm{~cm}$ \\
\hline Ferruginous & 1686 & 165 & $2 \%$ & $60 \%$ & $120 \mathrm{~cm}$ \\
\hline Woodland & 1411 & 5.3 & $5 \%$ & $3 \%$ & $120 \mathrm{~cm}$ \\
\hline Rock & NA & NA & NA & NA & $120 \mathrm{~cm}$ \\
\hline
\end{tabular}

\subsection{WATERTABLE CONTROL}

An important parameter is the soil moisture content because it affects electromagnetic and thermal soil properties and thus (possibly) the detection performance of the mine detection systems. In this test facility it is possible to regulate to some extent the soil moisture content of the top layer by controlling the groundwater level. For this reason the inside of every lane is covered with plastic to separate the groundwater in the lane from the water outside the lane. At the bottom of the 
lane a drainage tube is placed which is connected to a reservoir. The water level in the reservoirs, which are situated outside the $5 \mathrm{~m}$ zone which is made free of metals, is controlled automatically. The last column of Table 1 gives the values that have been used till now. In this way it is possible to test and evaluate the performance of detection systems under different moisture conditions, or to compare different systems under the same conditions. The actual groundwater level in the lanes can be checked at two points in every lane.

\section{MEASUREMENT PLATFORM}

A measurement platform has been developed which can carry individual sensors (TIR, MD and GPR) as well as a combination of sensors. Just like the test lanes, the measurement platform is made of materials that are not electrically conducting. It consists of a $17 \mathrm{~m}$ long glass fiber enforced polyester tube with a diameter of $0.9 \mathrm{~m}$. On top of this tube, which is shown in Figure 3, rails are constructed which are aligned to be flat within $5 \mathrm{~mm}$ over the full $17 \mathrm{~m}$. A sensor platform which can carry a total load of $600 \mathrm{~kg}$ moves automatically over these rails. The electric motor for this movement is positioned outside the zone which is made free of metals. The height of the sensors can be altered from $0 \mathrm{~cm}$ to 2 meter. Also the viewing angle of the sensors can be altered to view the surface from a different perspective. The polyester tube is moved from lane to lane manually over rails that are shown in Figure 2. In this direction (the east-west direction in Figure 2) it can be positioned with steps of $5 \mathrm{~cm}$. The reproducibility of the platform is $1 \mathrm{~cm}$ in all directions. The position of the sensor platform along the tube is measured continuously with a laser distance meter, and logged versus the time. This measurement has an accuracy of $2.5 \mathrm{~mm}$, and $50 \mathrm{~ms}$. These position values can be used to analyze the detection data afterwards.

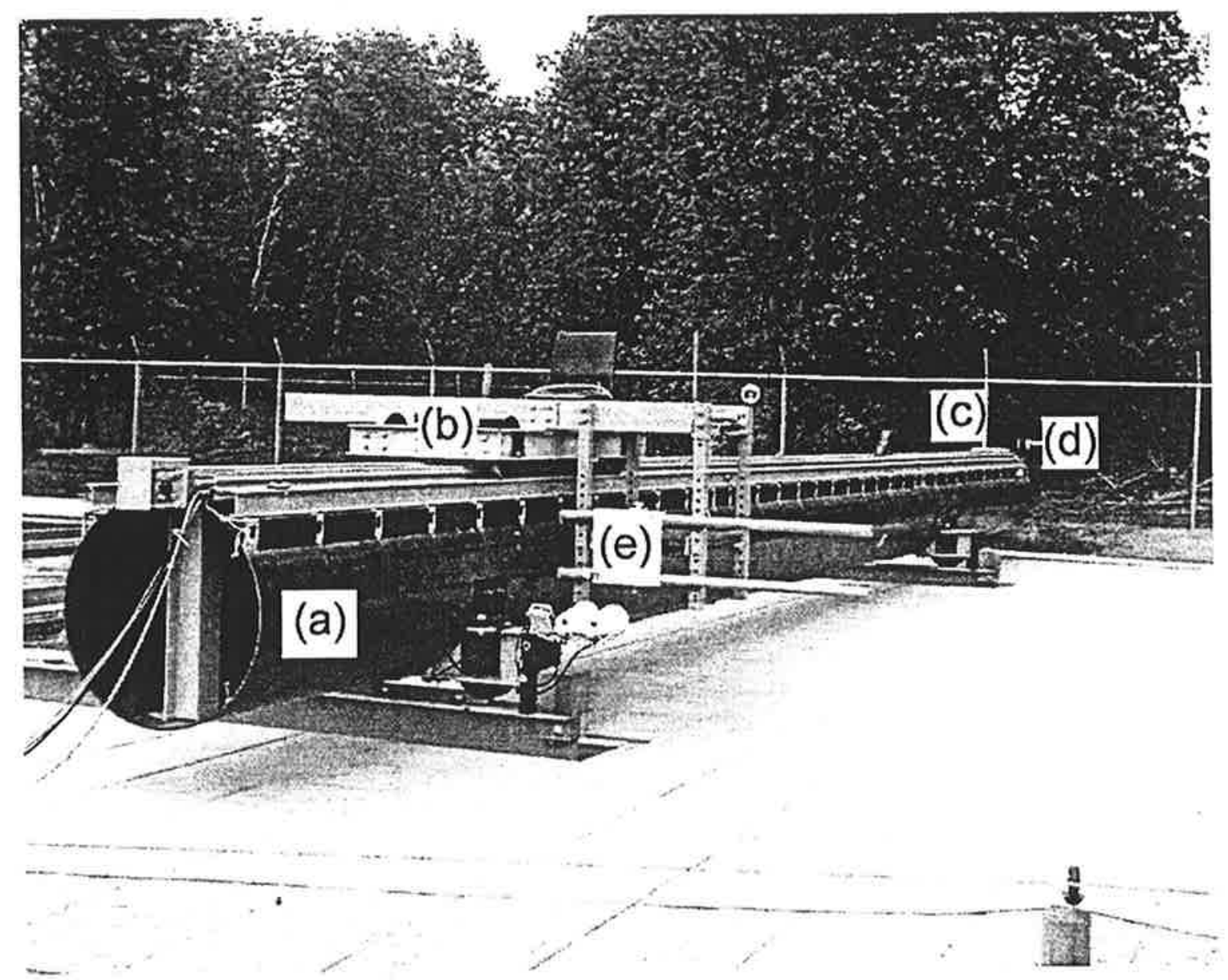

Figure 3. The measurement platform: (a) polyester tube. (b) sensor platform. (c) electric motor. (d) laser distance meter. (e) mount for a metal detector. The position of the measurement platform relative to the lanes is visible in Figure 1. 


\section{TEST MINES}

To perform the detection experiments, mines or minelike objects are needed. Since explosives in the test lanes at TNO are not allowed, live mines, or inert mines with explosives inside, can not be used in the lanes. To overcome this problem, test mines are used, having comparable signatures as real mines for the three types of sensors used. To cover the whole scenario of mines, both anti personnel (AP) and anti tank (AT) test mines were used, with the emphasis on anti personnel mines. Also some potential false alarms, like metal shrapnel of different sizes, aluminum cans, glass bottles, paving-stones and bricks were installed.

The AT and AP mines are divided in different categories:

- Metal mines, which have a metal casing or a high $(>20 \mathrm{~g})$ metal content.

- Medium metal content mines: having a non-metal casing and some metal $(10-20 \mathrm{~g})$ in the detonator.

- Low-metal mines: having a non-metal casing and a low $(<10 \mathrm{~g})$ metal content.

- Non-metal mines: these mines consist of material that is not electrically conducting.

Mines from all categories were used, with the emphasis on the mines with a medium or lower metal content, since metal mines are easily detectable with most metal detectors. In total about 350 minelike objects were used.

The signatures of mines are determined by different material properties. For detection with a metal detector or a ground penetrating radar system the electrical conductivity and the dielectric constant of both the casing and the interior of a mine determine its signature. These parameters, which are frequency dependent, should be comparable up to a frequency of $100 \mathrm{MHz}$. For a thermal infrared system, the temperature of the mine and the emission characteristics in the IR wavelength range determine its signature. The temperature of a buried mine depends on the heat capacity and the heat conductivity of all materials. If the mine is surface laid, also the solar absorption which is mainly in the visible range, and the IR absorption and emission influence the temperature.

\subsection{DIFFERENT TESTMINES}

According to the above mentioned requirements 7 different types of AP mines and 3 types of AT mines have been used (Table 2). Since it is difficult to acquire test mines that are realistic enough for the three detection methods, most of them were produced by TNO Physics and Electronics laboratory. To construct four mine types, drawings were used that originate from the Defence Science and Technology Organisation (DSTO) in Australia ${ }^{1}$. Three different mine casings were acquired from the Dutch army. The different materials for the casing show a variety, which is comparable to the variation that is found with real mines ${ }^{2-4}$. In all test mines the same substitute for the high explosive has been used (see next section).

Table 2. Different types of test mines that have been used.

\begin{tabular}{|l|l|l|}
\hline Type & casing material & metal content \\
\hline AP; type 1 & wood & medium \\
\hline AP; type 2 & plastic & high \\
\hline AP; type 3 & PVC & medium \\
\hline AP; type 4 & PVC & medium \\
\hline AP; type 5 & plastic & non \\
\hline AP; type 6 & ABS & low \\
\hline AP; type 7 & PE & medium \\
\hline & & \\
\hline AT; type 1 & metal & high \\
\hline AT; type 2 & PVC & low \\
\hline AT; type 3 & no casing & non \\
\hline
\end{tabular}



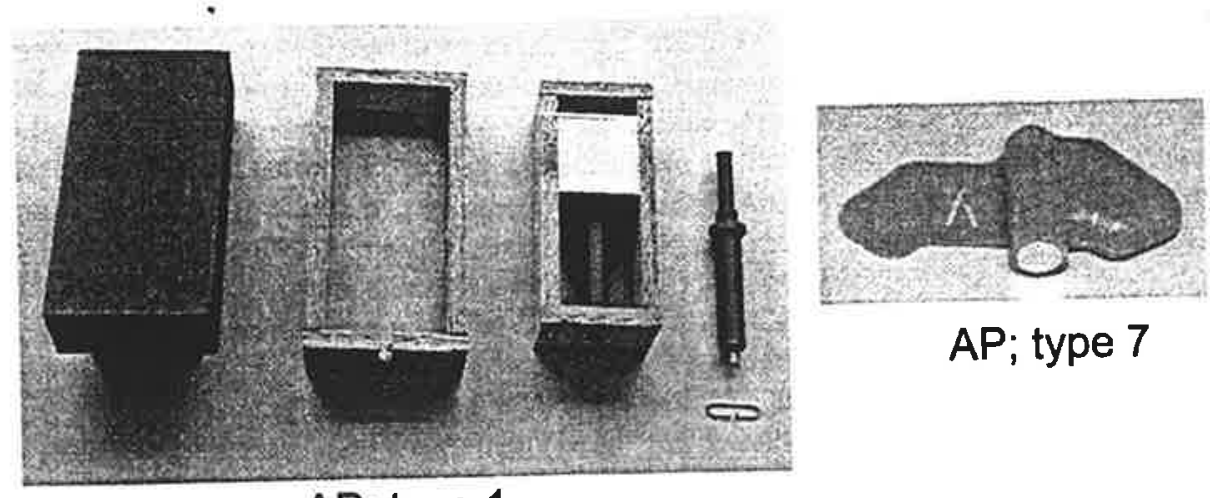

\section{AP; type 7}

\section{AP; type 1}
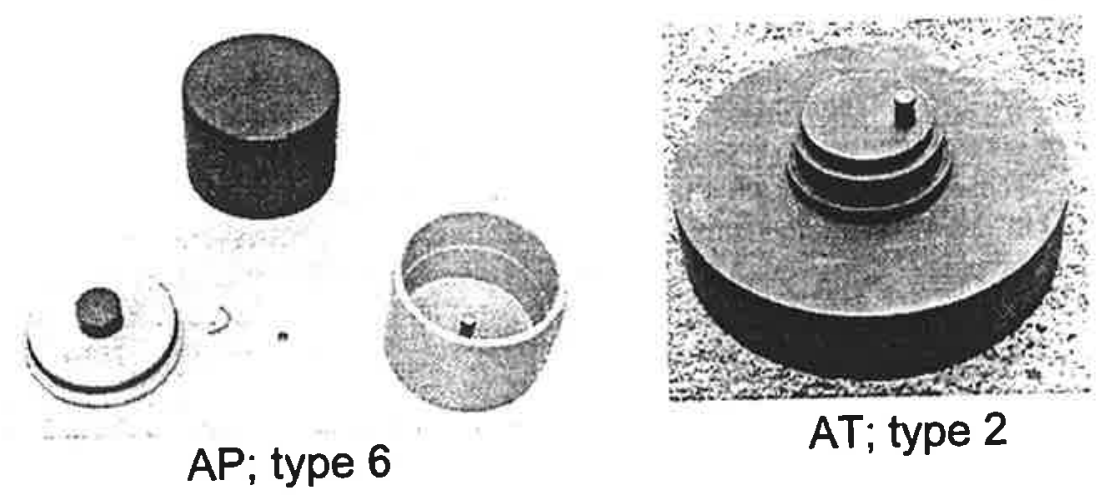

AT; type 2

Figure 4. Some test mines: From the AP type 1 and type 6 both the complete test mine and the separate parts are shown.

\subsection{FILLER MATERIAL}

A substitute for the high explosive is needed which has the same relevant characteristics as the high explosive. The most frequently used high explosives in mines are TNT, Composition B-3 (Comp B-3) and, less frequently, Tetryl ${ }^{2-4}$. A very suitable material is the silicone rubber RTV3110, produced by Dow Coming ${ }^{5-6}$. The properties of RTV3110, TNT, Comp B3 and Tetryl are summarized in Table 3.

Table 3. Properties of RTV3110 and some high explosives. The dielectric constant is measured at the frequency given in brackets.

\begin{tabular}{|l|c|c|c|c|}
\hline Properties & RTV3110 & TNT & Comp B-3 & Tetryl \\
\hline density $\left(\mathrm{g} / \mathrm{cm}^{3}\right)$ & $1,17^{5}$ & $1,56^{8}$ & $1,7^{8}$ & $1,7^{8}$ \\
\hline dielectric constant & $\begin{array}{c}2,90 \\
(1 \mathrm{MHz})^{5}\end{array}$ & $\begin{array}{c}2,88 \\
(5 \mathrm{MHz})^{7}\end{array}$ & & \\
\cline { 2 - 5 } & $\begin{array}{c}2,89 \\
(1 \mathrm{GHz})^{5}\end{array}$ & & $\begin{array}{c}3,25 \\
(3 \mathrm{GHz})^{7}\end{array}$ & $\begin{array}{c}3,10 \\
(35 \mathrm{GHz})^{7}\end{array}$ \\
\hline Heat capacity $(\mathrm{J} /(\mathrm{g} \mathrm{K}))$ & $1,50^{5}$ & $1,37^{8}$ & $1,25^{8}$ & $1,05^{8}$ \\
\hline Heat capacity $\left(\mathrm{J} /\left(\mathrm{cm}^{3} \mathrm{~K}\right)\right)$ & 1,76 & 2,14 & 2,13 & 1,79 \\
\hline Heat conductivity $(\mathrm{W} /(\mathrm{m} \mathrm{K}))$ & $0,20^{5}$ & $0,26^{8}$ & $0,219^{8}$ & $0,09^{8}$ \\
\hline
\end{tabular}

To check the thermal properties of RTV3110 versus those of TNT, cylinders of TNT and RTV3110 have been placed in a climate chamber. The cylinders were subjected to large changes in ambient temperature, for example a temperature drop of $60^{\circ} \mathrm{C}$ in less than three and a half hour (Figure 5a). The transient changes of the cylinder temperature were measured and 
compared. The results show very small temperature differences between the RTV3110 and the TNT (Figure 5b). Under less extreme conditions as exist in the TNO test facility, this difference is negligible. The electrical properties were not tested.
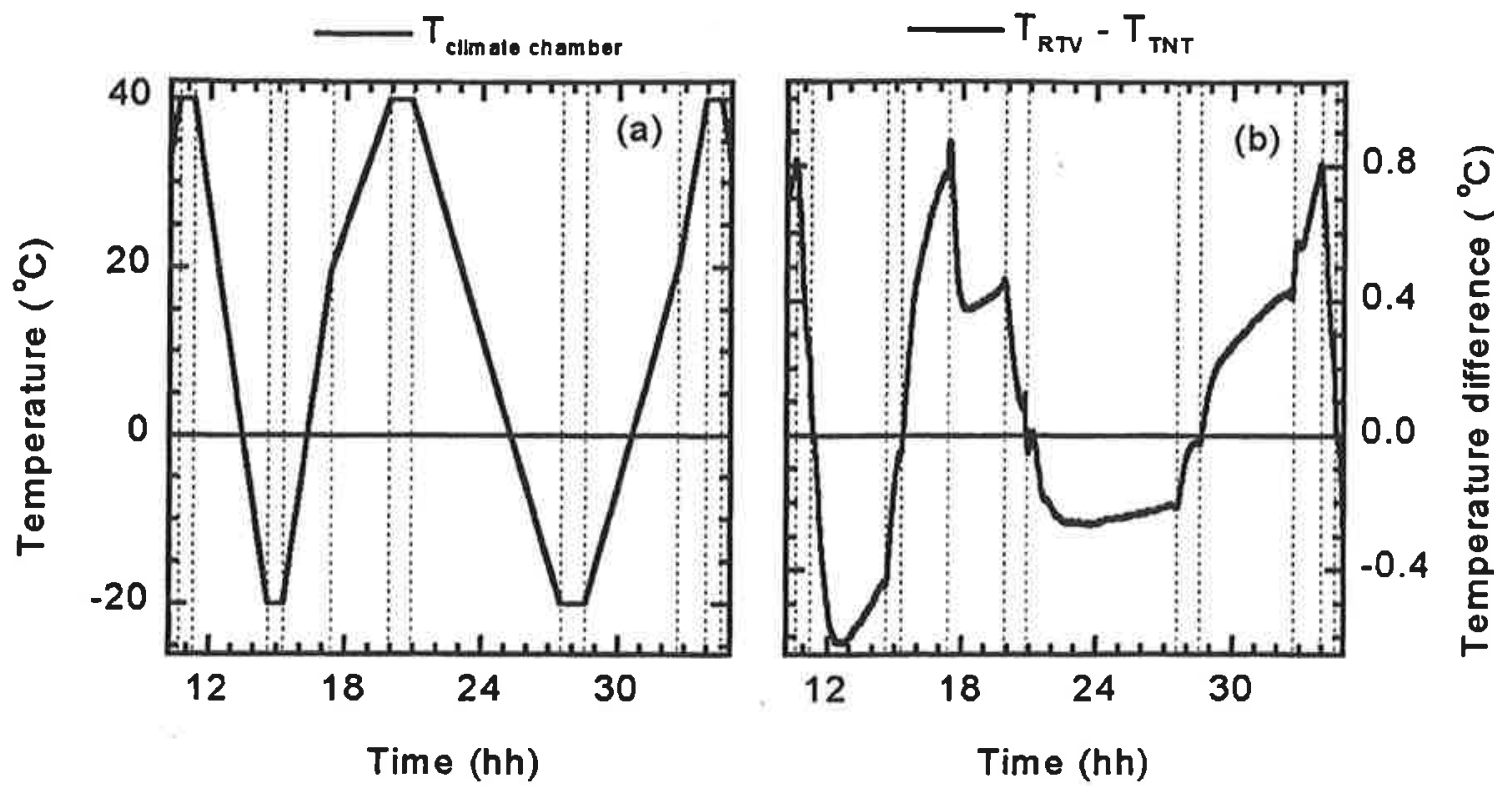

Figure 5. Figure a shows the temperature changes in the climate chamber. The vertical grid lines in this figure are drawn at the points where the derivative of the temperature changes. In Figure b the difference between the average temperature of the RTV3110 cylinders and the average temperature of the TNT cylinders is plotted. The grid lines are drawn at the same positions as in figure a. Note the difference in scale on the vertical axis.

\subsection{REFLECTION AND ABSORPTION PROPERTIES}

The diffuse reflection coefficients of the test mines have been measured for both the visible and the IR spectrum, up to $12 \mu \mathrm{m}$. Different types of real and test mines show different visible spectra as is shown in Figure 6 for a few types. This difference could be expected since the mines have different colors. However the spectra of a real mine and a test mine of the same type agree very well. In the IR wavelength bands the differences are much smaller and the spectra of the test mines agree quite well with those of real mines. In Table 4 the reflection coefficients are given for a few mines. 


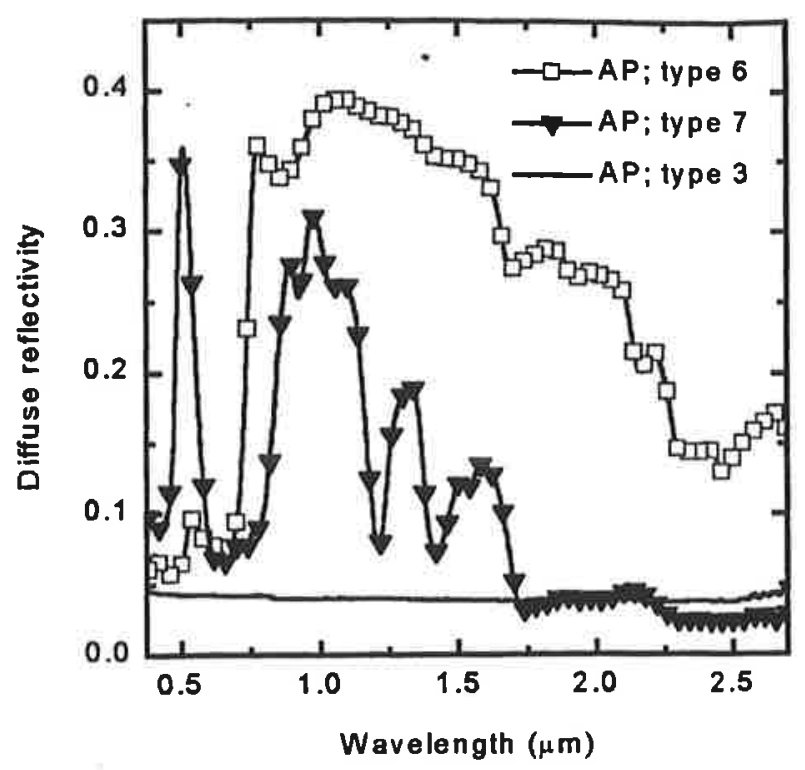

Figure 6 Reflectance spectra in the visible region of a few test mines.
Table 4. Diffuse reflection coefficients for two IR wavelength bands.

\begin{tabular}{|c|c|c|}
\hline $\begin{array}{c}\text { reflection } \\
3 \mu \mathrm{m}-6 \mu \mathrm{m}\end{array}$ & Test mine & Inert mine \\
\hline AP; type 3 & $0.07 \pm 0.02$ & $0.06 \pm 0.02$ \\
\hline AP; type 4 & $0.06 \pm 0.02$ & $0.06 \pm 0.02$ \\
\hline AP; type 6 & $0.09 \pm 0.02$ & $0.12 \pm 0.02$ \\
\hline AT; type 2 & $0.06 \pm 0.02$ & $0.06 \pm 0.02$ \\
\hline \hline & & \\
reflection & & \\
\hline Am- 13 $\mu \mathrm{m}$ & & \\
\hline AP; type 3 & $0.07 \pm 0.02$ & $0.03 \pm 0.02$ \\
\hline AP; type 4 & $0.04 \pm 0.02$ & $0.03 \pm 0.02$ \\
\hline AP; type 6 & $0.03 \pm 0.02$ & $0.03 \pm 0.02$ \\
\hline AT; type 2 & $0.05 \pm 0.02$ & $0.03 \pm 0.02$ \\
\hline
\end{tabular}

\subsection{CONFIGURATION OF TEST OBJECTS}

Each lane is divided in several parts as shown in Figure 7. This layout is in general identical for all lanes. In the empty reference part no objects are placed. This part can be used to measure the background level, if necessary. In the permanent parts test mines, summarized in Table 2 , and false alarms are laid in a structured pattern at different depths. The distance between an object and the walls of the lane and the distance between the objects is kept large enough to avoid disturbance of the detection of an object by one of its neighbors. This distance is larger for the AT test mines than for the AP test mines. This configuration is identical in the first 4 lanes. The lanes 5 and 6 have a slightly different pattem. Before the first detection tests started, the lanes were left to settle for two months to avoid the detection of disturbances in the soil due to the digging instead of the detection of a test mine. In the small IR, GPR and MD parts sensor specific objects can be placed like thermocouples or small metal objects at different depths. The variable part is reserved for placing test objects ad-hoc without disrupting the permanently laid mines. All relevant data concerning the test mines and false alarms is stored in a database.

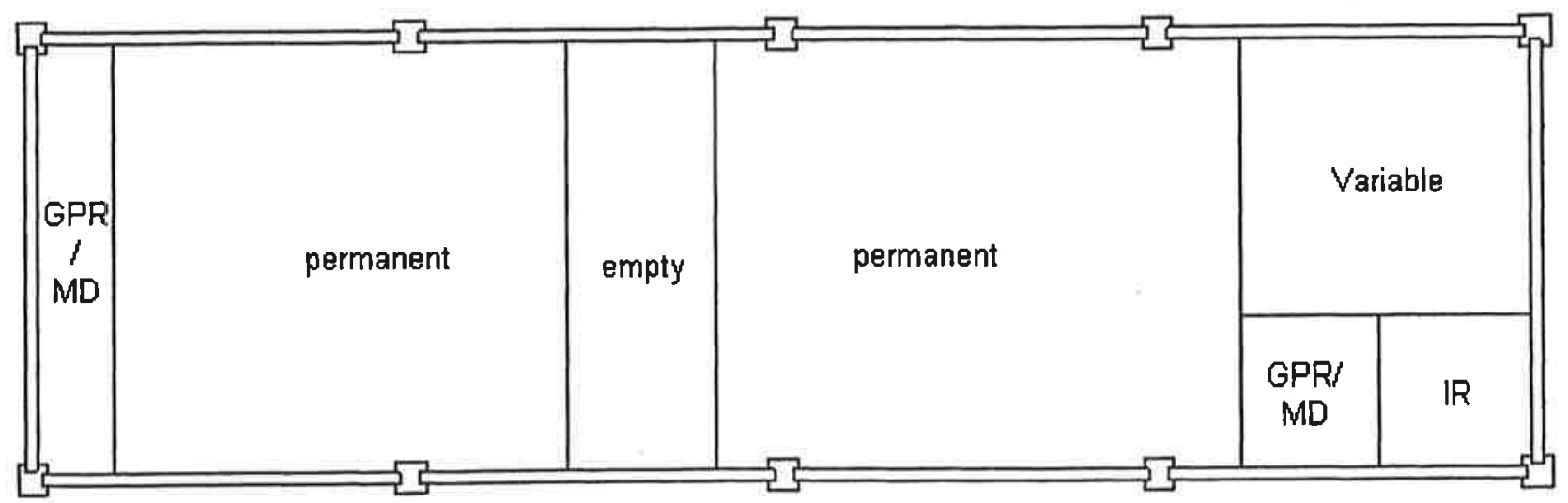

Figure 7. Layout of the first four lanes. 


\section{ADDITIONAL FACILITIES}

To evaluate the detection results correctly, additional data is gathered.

The weather conditions are measured and recorded. Two meteo stations, one at ground level and one at a height of $3 \mathrm{~m}$ have been set up to measure the standard meteorological observations that are: global radiation, air temperature, relative humidity, wind speed, wind direction and precipitation. The soil moisture was regularly measured in the variable part of every lane with a time domain reflectometer (TDR).

In the IR part (Figure 7) of every lane a few mines of different types and at different depths are equipped with thermocouples. The temperatures of these test mines and a soil profile till a depth of $30 \mathrm{~cm}$ are continuously monitored. This information is especially useful for evaluating the detection results of thermal infrared systems. Figure 8 gives the results of temperature measurements in the lane filled with sand.

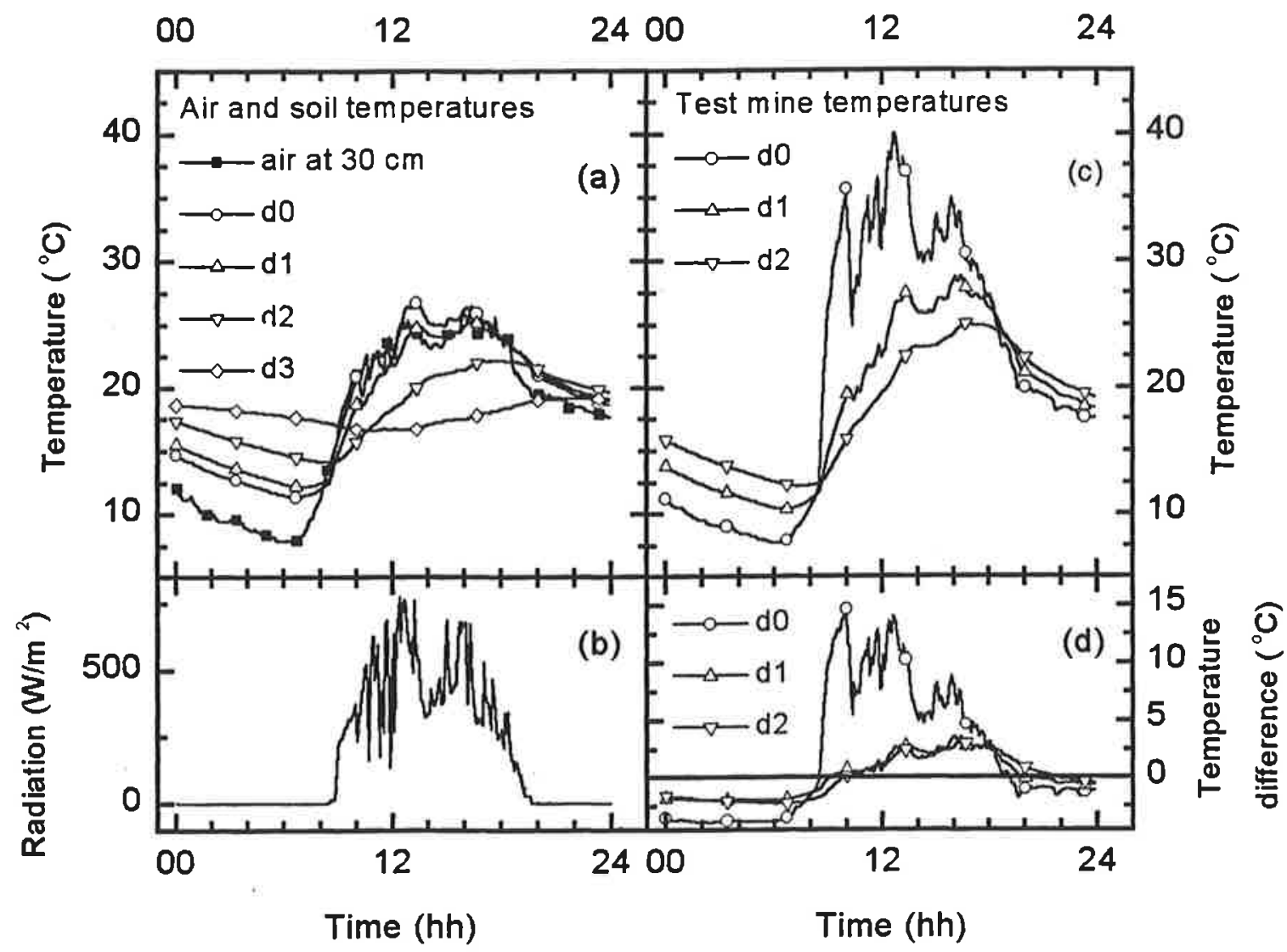

Figure 8. Results of temperature measurements in the lane filled with sand. Figure a gives the temperature measured with the meteo station $30 \mathrm{~cm}$ above the surface, and the temperature at 4 different depths, d0 is at the surface, d1 $=2.5 \mathrm{~cm}$ below surface, $d 2=10 \mathrm{~cm}$ below surface and $d 3=30 \mathrm{~cm}$ below surface. The global radiation ( $0.3 \mu \mathrm{m}$ to $2.8 \mu \mathrm{m})$ is plotted in figure $b$. In figure $c$ the temperatures of a small AP mine buried at different depths is given. The difference between the temperature of the mines and the soil at the same depth is given in figure $d$.

For protection of the test facility, an advanced security system for detection of intruders and small game, has been put on the whole testing area. Before installation of this system, small game was digging up mines. 


\section{DISCUSSION}

With use of the described test facility measurements with the individual sensors (TIR, MD and GPR) and with a combined system of sensors have been performed. Results of these tests are presented in references 9 en 10. During these experiments it became clear that a facility with well defined conditions is absolutely necessary to interpret the results and to compare the results of different sensors.

Up to now the test facility has been used for the HOM-2000 project only. However discussions are in progress with other possible users.

\section{ACKNOWLEDGEMENTS}

The authors are grateful for the fruitful discussions and collaboration with P.J. Fritz, A. Schoolderman, A. Wilbers, J. Sluiter, N.L.P. de Bruyn Prince van Kempen and all other participants in the HOM-2000 project. The HOM-2000 project is performed under contract with the Dutch ministry of Defense.

\section{REFERENCES}

1. Benny C.Y. Wong, Ian Chant, Graeme N. Crisp, Karl Kappra, Keith Sturgess, Alay Rye, and Kelly Sherbondy, "Suggested soil characterization techniques and surrogate targets for ultra-wide-band radar mine detection experiments", in Detection and Remediation Technologies for Mines and Mlinelike Targets II, Abinash C. Dubey, Robert L. Barnard, Editors, Proceedings of SPIE Vol. 3079, 555-567, (1997). Figures 8 to 11.

2. Department of Defense, MineFacts, Department of Defense, Washington, USA, 1995, CD-ROM

3. R.A. MacDougall, The CFSME Mine Database 96, CSFME, Chiliwack, Canada, 1996, CD-ROM.

4. Colin King, editor, Jane's mines and mine clearance, Jane's, Coulsdon, Surrey, UK, 1996.

5. Product Information for Silastic 3110 RTV Silicone Rubber, Dow Corning Europe, Brussels, 1981, Data Sheet number 61-143-01.

6. B. Scheers, M. Piette, and A. Vander Vorst, "The Detection of AP Mines using UWB GPR", in The detection of abandoned land mines, Conference Publication No. 458 IEE, 45-49, 1998.

7. D.L. Patel, Handbook of Land Mines and Military Explosives for Countermine Exploitation, US Army, Belvoir Research, Development and Engineering Center, Fort Belvoir, Virginia, 1992, US Army report, 2495.

8. B.M. Dobratz and P.C. Crawford, LLNL Explosives Handbook; Properties of Chemical Explosives and Explosive Simulants, Lawrence Livermore National Laboratory; University of California, Livermore, California, 1985, UCRL52997.

9. M.G.J. Breuers, P.B.W. Schwering, S.P. van den Broek, "Sensor fusion algorithms for the detection of land mines", in Detection and Remediation Technologies for Mines and Minelike Targets IV, Proceedings of SPIE Vol. 3710, (1999) to be published.

10. J.B. Rhebergen, A.P.M. Zwamborn, and D.V. Giri, "Design of an ultra-wideband ground-penetrating radar system using impulse radiating antennas", in The detection of abandoned land mines, Conference Publication No. 458 IEE, $45-49,1998$. 